\title{
PHASE SPACE HEISENBERG-LIMITED ESTIMATION OF THE AVERAGE PHASE SHIFT IN A MACH-ZEHNDER INTERFEROMETER
}

\author{
DARIO GATTO \\ School of Mathematics and Physics, University of Portsmouth, \\ Portsmouth, Hampshire PO1 3QL, United Kingdom \\ dario.gatto@port.ac.uk \\ PAOLO FACCHI \\ Dipartimento di Fisica and MECENAS, Università di Bari, I-70126 Bari, Italy, \\ INFN, Sezione di Bari, I-70126 Bari, Italy \\ VINCENZO TAMMA \\ School of Mathematics and Physics, University of Portsmouth, \\ Portsmouth, Hampshire PO1 3QL, United Kingdom \\ Institute of Cosmology and Gravitation, University of Portsmouth, \\ Portsmouth, Hampshire PO1 3QL, United Kingdom \\ vincenzo.tamma@port.ac.uk \\ Received Day Month Year \\ Revised Day Month Year
}

\begin{abstract}
We address the problem of distributed quantum metrology with a single squeezedvacuum source by using the formalism of quantum mechanics in phase space. In particular we demonstrate Heisenberg-limited sensitiviy in the measurement of the average of two arbitrary phase shifts in the arms of a Mach-Zehnder interferometer. We obtain exact results for the measurement probability at the interferometer output for any value of the phases, which give us insight into the emergence of Heisenberg-limited sensitivity for periodical values of the phases.
\end{abstract}

Keywords: Quantum metrology; Heisenberg limit; Quantum optics; Squeezed states.

\section{Introduction}

Quantum metrology is one of the most promising emergent quantum technologies, offering the possibility to boost and overcome the limitations of classical physics, in devising measurement schemes with a sensitivity far superior to what is fundamentally possible without employing quantum resources ${ }^{1}\left[\frac{1}{8}\right.$ In particular, the problem of measuring several parameters distributed over a linear optical network has recently sparked much interest in the research community 9914 Possible applications range from the mapping of inhomogenous magnetic fields, $\frac{15-19}{19}$ phase imaging $20 \mid 25$ 
quantum-enhanced nanoscale nuclear magnetic resonance imaging,$\underline{926}[27$ to applications in precision clocks, ${ }^{28}$ geodesy, and geophysics $\stackrel{29}{21}$

In a previous work ${ }^{32}$ we have proposed the use of squeezed light as a resource for overcoming the technological limitations of the state-of-the-art interferomenter for the estimation of a linear combination of $M>1$ unknown parameters, ${ }^{33}$ and achieving Heisenber-limited estimation sensitivity, i.e. a sensitivity scaling as $1 / \bar{N}$, where $\bar{N}$ is mean number of photons in the interferometer. It was later shown that our configuration is in fact optimal ${ }^{34}$ In this article, we revisit the $M=2$ scenario using the formalism of quantum mechanics in phase space. This allows us to obtain exact results, resulting in a much more intuitive and satisfying physical picture. Our aim will be to estimate the average phase shift in a 2-channel interferometer with a sensitivity scaling at Heisenberg limit.

\section{Theoretical description of the interferometer}

Let us consider a balanced Mach-Zehnder interferometer (see the schematic in Fig. (1)) where one of the input channels is fed with a squeezed vacuum state, characterized by the squeezing parameter $z=r e^{2 i \theta}$, and the other channel is left in the vacuum state. The two-mode state $\hat{\rho}$ at the input, as well as at any stage of

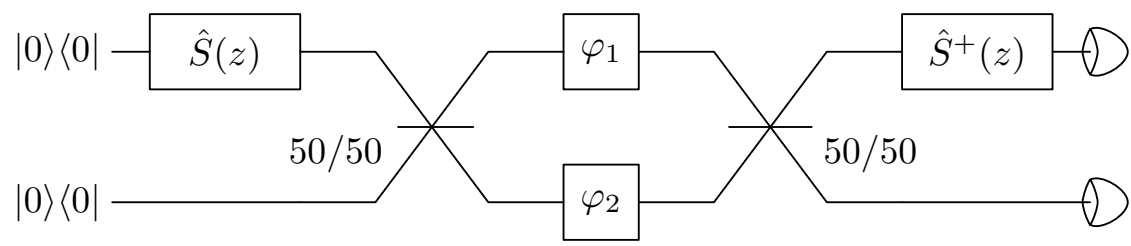

Fig. 1. Schematic of the interferometer for the estimation of the average phase shift $\left(\varphi_{1}+\varphi_{2}\right) / 2$. The interferometer is a balanced Mach-Zehnder configuration preceded by a squeezing operation, described by $\hat{S}(z)=e^{\frac{1}{2}\left(z \hat{a}^{\dagger 2}-z^{*} \hat{a}^{2}\right)}$, where $z$ is the squeezing parameter and $\hat{a}$ is the bosonic annihilation operator on either channel, and followed by an anti-squeezing operation $\hat{S}^{\dagger}(z)$. Onoff photodetectors are placed at the end of the interferometer.

the interferometer, is a Gaussian state described by a Gaussian Wigner function ${ }^{355}$

$$
W_{\hat{\rho}}(\boldsymbol{\xi})=\frac{\mathrm{e}^{-\frac{1}{2} \boldsymbol{\xi}^{T} \sigma^{-1} \boldsymbol{\xi}}}{(2 \pi)^{2} \sqrt{\operatorname{det} \sigma}}
$$

where $\boldsymbol{\xi}=\left(x_{1}, p_{1}, x_{2}, p_{2}\right)^{T}$ is the two-mode phase-space coordinate, $\hat{\mathbf{R}}=$ $\left(\hat{X}_{1}, \hat{P}_{1}, \hat{X}_{2}, \hat{P}_{2}\right)^{T}$ is the field quadratures vector operator, and $\sigma=\left(\frac{1}{2}\left\langle\hat{R}_{j} \hat{R}_{k}+\right.\right.$ $\left.\left.\hat{R}_{k} \hat{R}_{j}\right\rangle\right)(1 \leq j, k \leq 4)$ is the covariance matrix. In the vacuum state, $\hat{\rho}_{\text {vac }}=|00\rangle\langle 00|$, one gets $\sigma_{\mathrm{vac}}=I_{4} / 2$, while at the input of the interferometer, after the action of the one-mode squeezing operation in Fig. (1), the covariance matrix reads

$$
\sigma_{\mathrm{in}}=R^{T} Q \sigma_{\mathrm{vac}} Q R=\frac{1}{2} R^{T} Q^{2} R,
$$


where 35

$$
Q=\left(\begin{array}{cccc}
e^{r} & 0 & 0 & 0 \\
0 & e^{-r} & 0 & 0 \\
0 & 0 & 1 & 0 \\
0 & 0 & 0 & 1
\end{array}\right), \quad R=\left(\begin{array}{cccc}
\cos \theta-\sin \theta & 0 & 0 \\
\sin \theta & \cos \theta & 0 & 0 \\
0 & 0 & 1 & 0 \\
0 & 0 & 0 & 1
\end{array}\right)
$$

Therefore, the mean photon number of the state is $\bar{N}=\frac{1}{2} \operatorname{tr}\left(\sigma_{\text {in }}\right)-1=\frac{1}{4} \operatorname{tr}\left(Q^{2}\right)-1=$ $\sinh ^{2}(r)$.

The evolution of the probe state through the interferometer is described by the covariance matrix transformation

$$
\sigma_{\mathrm{MZ}}=O_{\mathrm{MZ}} \sigma_{\mathrm{in}} O_{\mathrm{MZ}}^{T}
$$

where the orthogonal and symplectic matrix $O_{\mathrm{Mz}}$ describing the Mach-Zehnder interferometer reads

$$
O_{\mathrm{MZ}}=\left(\begin{array}{cccc}
\cos \varphi_{+} \cos \varphi_{-} & -\sin \varphi_{+} \cos \varphi_{-} & -\sin \varphi_{+} \sin \varphi_{-} & -\cos \varphi_{+} \sin \varphi_{-} \\
\sin \varphi_{+} \cos \varphi_{-} & \cos \varphi_{+} \cos \varphi_{-} & \cos \varphi_{+} \sin \varphi_{-} & -\sin \varphi_{+} \sin \varphi_{-} \\
-\sin \varphi_{+} \sin \varphi_{-} & -\cos \varphi_{+} \sin \varphi_{-} & \cos \varphi_{+} \cos \varphi_{-} & -\sin \varphi_{+} \cos \varphi_{-} \\
\cos \varphi_{+} \sin \varphi_{-} & -\sin \varphi_{+} \sin \varphi_{-} & \sin \varphi_{+} \cos \varphi_{-} & \cos \varphi_{+} \cos \varphi_{-}
\end{array}\right),
$$

where $\varphi_{ \pm}=\left(\varphi_{1} \pm \varphi_{2}\right) / 2, \varphi_{1}$ is the phase shift accumulated by the probe on the first channel, and $\varphi_{2}$ is the phase shift accumulated by the probe on the second channel (see Appendix for a derivation).

The probe is successively exposed to an anti-squeezing operation, which is associated with the covariance matrix transformation

$$
\sigma_{\mathrm{out}}=Q^{-1} R \sigma_{\mathrm{MZ}} R^{T} Q^{-1},
$$

where $Q$ and $R$ are defined in Eq. (3). Finally, on-off photodetectors are placed at the output of the interferometer.

\section{Detection probability and estimation sensitivity}

The probability is $P=\operatorname{tr}\left(|00\rangle\langle 00| \hat{\rho}_{\text {out }}\right)$ of measuring the vacuum in both channels of the interferometer, reads

$$
P=\pi^{2} \int W_{\hat{\rho}_{\text {out }}}(\boldsymbol{\xi}) W_{\hat{\rho}_{\text {vac }}}(\boldsymbol{\xi}) \mathrm{d}^{4} \xi=\int \frac{\mathrm{e}^{-\frac{1}{2} \boldsymbol{\xi}^{T}\left(\sigma_{\text {out }}^{-1}+\sigma_{\text {vac }}^{-1}\right) \boldsymbol{\xi}}}{(2 \pi)^{2} \sqrt{\operatorname{det} \sigma_{\text {out }}}} \mathrm{d}^{4} \xi,
$$

where $\hat{\rho}_{\text {out }}$ is the Gaussian state associated with the covariance matrix (6), while $\sigma_{\text {vac }}=I / 2$. By performing the Gaussian integration we obtain the exact expression of the measurement probability

$$
\begin{aligned}
P= & {\left[1-\cos ^{2} \varphi_{-} \cos \left(2 \varphi_{+}\right) \bar{N}\right.} \\
& \left.\quad-\left(1+\cos ^{2} \varphi_{-} \frac{1}{4} \sin ^{2}\left(2 \varphi_{-}\right)-2 \cos ^{2} \varphi_{-} \cos \left(2 \varphi_{+}\right)\right) \frac{\bar{N}^{2}}{4}\right]^{-1 / 2} .
\end{aligned}
$$




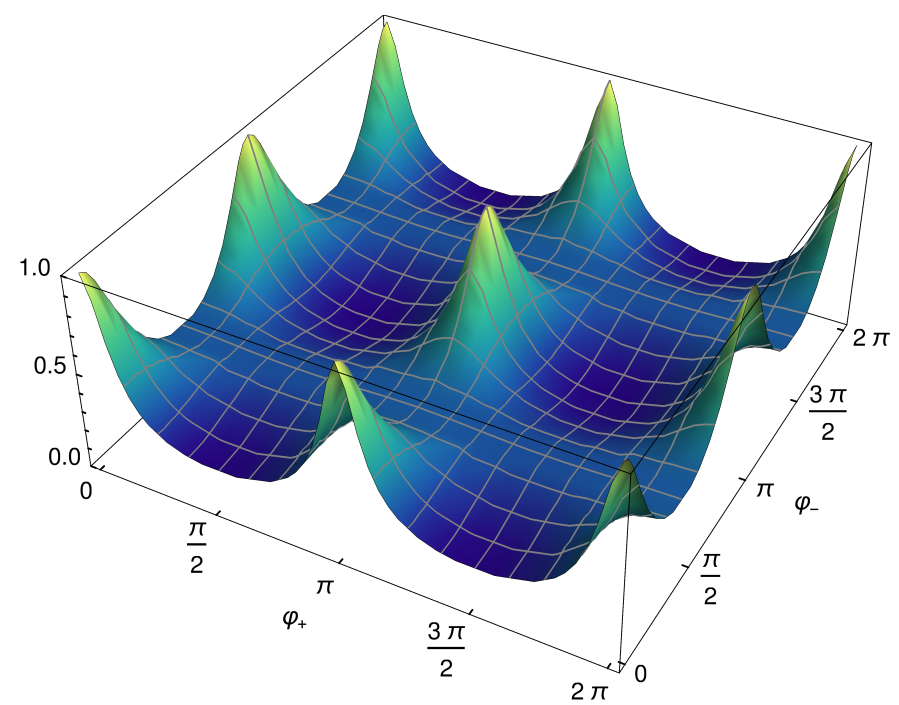

(a) $\bar{N}=4$

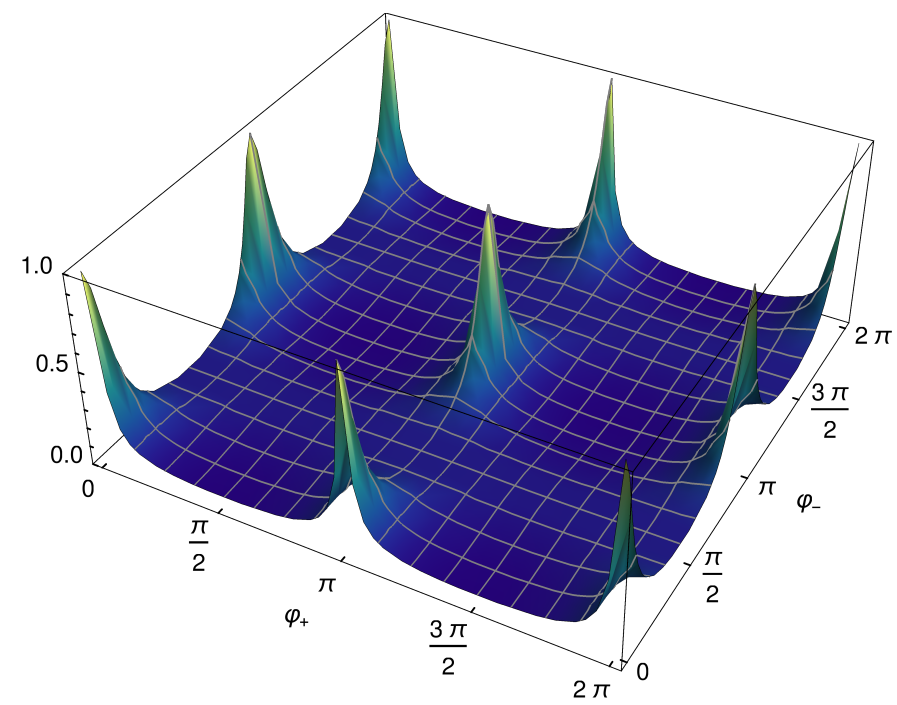

(b) $\bar{N}=20$

Fig. 2. Detection probability as a function of $\varphi_{ \pm}=\left(\varphi_{1} \pm \varphi_{2}\right) / 2$ for various mean photon numbers. The peaks concentrate way quickly on the $\varphi_{+}$-direction than they do on the $\varphi_{+}$-direction, becoming more and more localized as $\bar{N}$ increases, which is a crucial ingredient for Heisenberg scaling.

The most striking feature of this quantity is the fact that both $\varphi_{-}$and $\varphi_{+}$affect the detection probability, as can be easily seen from the plot in Fig. (2). As discussed in Ref. 32, this "unusual" phenomenon, where it is not only the relative phase shift between the two channels of the interferometer that bears a physical significance, 
but rather both phase shifts do, is actually the general case in quantum interferometry and should be expected in this particular scheme. Another important aspect is the periodicity of the probability in $\varphi_{+}$and $\varphi_{-}$up to multiples of $\pi$, which was not evident in the approximated approach carried out in Ref. 32 .

We shall now estimate the sensitivity associated with this scheme. Formally, the quantum observable measured at the output of the interferometer is the projection on the vacuum $\hat{\Pi}=|00\rangle\langle 00|$, and

$$
\langle\hat{\Pi}\rangle=P,
$$

where the expectation value is taken on the quantum state at the output of the interferometer. By the error propagation formula, the sensitivity of the scheme can be estimated as

$$
\Delta \varphi_{ \pm}^{2}=\frac{\left\langle\hat{\Pi}^{2}\right\rangle-\langle\hat{\Pi}\rangle^{2}}{\left|\partial\langle\hat{\Pi}\rangle / \partial \varphi_{ \pm}\right|^{2}}=\frac{P(1-P)}{\left|\partial P / \partial \varphi_{ \pm}\right|^{2}}
$$

It is evident from this expression and the scaling of Eq. (8) with $\bar{N}$ that there cannot be Heisenberg-limited sensitivity for every value of $\varphi_{+}$and $\varphi_{-}$. However, Heisenberg scaling is possible where $P \simeq 1$, i.e. for phases in a neighbourhood of one of the maxima in Fig. (2). This suggests constructive quantum interference is in fact a metrological resource. Hence, for phases $\left(\varphi_{+}, \varphi_{-}\right)$in a small neighbourhood of a maximum $\left(k_{+} \pi, k_{-} \pi\right)$ with integers $k_{ \pm}$, Eq. (8) reduces to

$$
P \simeq 1+2\left(\bar{N}^{2}+\bar{N}\right)\left(\varphi_{+}-k_{+} \pi\right)^{2}+\bar{N}\left(\varphi_{-}-k_{-} \pi\right)^{2}
$$

for $\left|\varphi_{ \pm}-k_{ \pm} \pi\right| \ll 1 / \bar{N}$.

When the mean photon number is large we can retain only the leading terms in $\bar{N}$, leading us to a simple expression depending only on the average phase shift we wish to estimate,

$$
P \simeq 1+2 \bar{N}^{2}\left(\varphi_{+}-k_{+} \pi\right)^{2}
$$

The fact that the dominant contribution depends only on $\varphi_{+}$could have been anticipated by noticing that, as $\bar{N}$ increases, the variation of $P$ in the neighbourhood of a maximum is much more stark and rapid along the $\varphi_{+}$axis than it is on the $\varphi_{-}$axis, where it is almost constant in comparison (see again Fig. 2p). This is a reflection of the localization along the $\varphi_{+}$-direction of constructive quantum interference, and is important for reaching Heisenberg-limited sensiivity. Therefore, by Eq. 10 , the estimation sensitivity reads

$$
\Delta \varphi_{+}^{2} \simeq \frac{1}{8 \bar{N}^{2}}
$$

which exhibits the scaling typical of the Heisenberg limit. 


\section{Conclusions}

We have revisited the protocol for distributed quantum metrology introduced in Ref. 32 in the case of a simple Mach-Zehnder configuration with a single squeezedstate source and an anti-squeezing operation at one of the interferometer output channels. In particular, we have exploited the formalism of quantum mechanics in phase space to obtain an exact result for probability of measuring the vacuum in both output channels. This has allowed us to demonstrate Heisenberg-limited sensitivity for the measurement of the average phase shift in the interferometer for periodic values where the measurement probability is maximal. These results give us insight on the role of constructive quantum interference in distributed quantum metrology, which we will further explore in future works.

\section{Acknowledgments}

This project was partially supported by the Office of Naval Research (ONR) Global (Award No. N62909-18-1-2153). DG is supported by the University of Portsmouth. P.F. is partially supported by Istituto Nazionale di Fisica Nucleare (INFN) through the project "QUANTUM," and by the Italian National Group of Mathematical Physics of Istituto Nazionale di Alta Matematica (GNFM-INdAM).

\section{Appendix. Linear optical networks in phase space}

A passive linear optical network is described by a unitary operator $\hat{U}$ acting on the underlying Hilbert space of an $M$-mode electromagnetic field. Its action on the annihilation operators is

$$
\hat{U}^{\dagger} \hat{a}_{i} \hat{U}=\sum_{j=1}^{M} \mathcal{U}_{i j} \hat{a}_{j} .
$$

Let $\hat{\mathbf{R}}=\left(\hat{X}_{1}, \hat{P}_{1}, \ldots, \hat{X}_{M}, \hat{P}_{M}\right)^{T}$. Defining the unitary $2 M \times 2 M$ unitary matrix

$$
W=\frac{1}{\sqrt{2}} \bigoplus_{i=1}^{M}\left(\begin{array}{cc}
1 & i \\
1 & -i
\end{array}\right),
$$

we have the identity

$$
\hat{\mathbf{A}}:=\left(\hat{a}_{1}, \hat{a}_{1}^{\dagger}, \ldots, \hat{a}_{M}, \hat{a}_{M}^{\dagger}\right)^{T}=W \hat{\boldsymbol{R}}
$$

Thus,

$$
\begin{aligned}
\hat{U}^{\dagger} \hat{R}_{i} \hat{U} & =\hat{U}^{\dagger} \sum_{j=1}^{M}\left(W_{i, 2 j-1}^{\dagger} \hat{a}_{j}+W_{i, 2 j}^{\dagger} \hat{a}_{j}^{\dagger}\right) \hat{U}=\sum_{j, k=1}^{M}\left(W_{i, 2 j-1}^{\dagger} \mathcal{U}_{j k} \hat{a}_{k}+W_{i, 2 j}^{\dagger} \mathcal{U}_{j k}^{*} \hat{a}_{k}^{\dagger}\right)= \\
& =\sum_{h=1}^{2 M} \sum_{j, k=1}^{M}\left(W_{i, 2 j-1}^{\dagger} \mathcal{U}_{j k} W_{2 k-1, h}+W_{i, 2 j}^{\dagger} \mathcal{U}_{j k}^{*} W_{2 k, h}\right) \hat{R}_{h}:=\sum_{h=1}^{2 M} O_{i h} \hat{R}_{h} .
\end{aligned}
$$


The matrix $O$ can hence be represented as

$$
O=W^{\dagger}\left(\begin{array}{cccccc}
\mathcal{U}_{11} & 0 & \ldots & \ldots & \mathcal{U}_{1 M} & 0 \\
0 & \mathcal{U}_{11}^{*} & \ldots & \ldots & 0 & \mathcal{U}_{1 M}^{*} \\
\vdots & \vdots & \ddots & \ddots & \vdots & \vdots \\
\vdots & \vdots & \ddots & \ddots & \vdots & \vdots \\
\mathcal{U}_{M 1} & 0 & \ldots & \ldots & \mathcal{U}_{M M} & 0 \\
0 & \mathcal{U}_{M 1}^{*} & \ldots & \ldots & 0 & \mathcal{U}_{M M}^{*}
\end{array}\right) W
$$

where $W$ has been defined in Eq. 15). As can easily be verified, this matrix is both orthogonal and symplectic, and can be simplified as follows

$$
O=\left(\begin{array}{cccccc}
\operatorname{Re}\left(\mathcal{U}_{11}\right) & -\operatorname{Im}\left(\mathcal{U}_{11}\right) & \ldots & \ldots & \operatorname{Re}\left(\mathcal{U}_{1 M}\right) & -\operatorname{Im}\left(\mathcal{U}_{1 M}\right) \\
\operatorname{Im}\left(\mathcal{U}_{11}\right) & \operatorname{Re}\left(\mathcal{U}_{11}\right) & \ldots & \ldots & \operatorname{Im}\left(\mathcal{U}_{1 M}\right) & \operatorname{Re}\left(\mathcal{U}_{1 M}\right) \\
\vdots & \vdots & \ddots & \ddots & \vdots & \vdots \\
\vdots & \vdots & \ddots & \ddots & \vdots & \vdots \\
\operatorname{Re}\left(\mathcal{U}_{M 1}\right) & -\operatorname{Im}\left(\mathcal{U}_{11}\right) & \ldots & \ldots & \operatorname{Re}\left(\mathcal{U}_{11}\right) & -\operatorname{Im}\left(\mathcal{U}_{11}\right) \\
\operatorname{Im}\left(\mathcal{U}_{M 1}\right) & \operatorname{Re}\left(\mathcal{U}_{M 1}\right) & \ldots & \ldots & \operatorname{Im}\left(\mathcal{U}_{11}\right) & \operatorname{Re}\left(\mathcal{U}_{M M}\right)
\end{array}\right)
$$

Therefore, the action of passive linear optical network on a Gaussian state with covariance matrix $\sigma_{\text {in }}=\frac{1}{2}\left\langle\hat{\boldsymbol{R}} \hat{\boldsymbol{R}}^{T}\right\rangle+\frac{1}{2}\left\langle\hat{\boldsymbol{R}} \hat{\boldsymbol{R}}^{T}\right\rangle^{T}$ is simply the transformation

$$
\sigma_{\text {out }}=O \sigma_{\text {in }} O^{T}
$$

that is, nothing but a (symplectic) rotation in phase space. In the case of the Mach-Zehnder interferometer, the $\mathcal{U}$ matrix reads

$$
\mathcal{U}_{\mathrm{MZ}}=\frac{1}{2}\left(\begin{array}{cc}
1 & -i \\
-i & 1
\end{array}\right)\left(\begin{array}{cc}
e^{i \varphi_{1}} & 0 \\
0 & e^{i \varphi_{2}}
\end{array}\right)\left(\begin{array}{ll}
1 & i \\
i & 1
\end{array}\right)=e^{i \varphi_{+}}\left(\begin{array}{cc}
\cos \varphi_{-} & -\sin \varphi_{-} \\
\sin \varphi_{-} & \cos \varphi_{-}
\end{array}\right),
$$

from which Eq. (5) in the text follows.

\section{References}

1. C. M. Caves, Quantum-mechanical noise in an interferometer, Phys. Rev. D 23, 1693 (1981).

2. B. Yurke, S. L. McCall, and J. R. Klauder, SU(2) and SU(1,1) interferometers, Phys. Rev. A 33, 4033 (1986).

3. M. J. Holland and K. Burnett, Interferometric detection of optical phase shifts at the Heisenberg limit, Phys. Rev. Lett. 71, 1355 (1993).

4. V. Giovannetti, S. Lloyd, and L. Maccone, Quantum-enhanced measurements: beating the standard quantum limit, Science 306, 1330 (2004).

5. V. Giovannetti, S. Lloyd, and L. Maccone, Quantum Metrology, Phys. Rev. Lett. 96, 010401 (2006).

6. V. Giovannetti, S. Lloyd, and L. Maccone, Advances in Quantum Metrology, Nature Photonics 5, 222 (2011).

7. L. Pezzè and A. Smerzi, in Proceedings of the International School of Physics "Enrico Fermi", Course 188 (IOS Press, Amsterdam, 2014), p. 691. 
8. R. Demkowicz-Dobrazánski, M. Jarzyna, and J. Kołodyński, Quantum Limits in Optical Interferometry, Progress in Optics 60, 345 (2015).

9. Z. Eldredge, M. Foss-Feig, J. A. Gross, S. L. Rolston, and A. V. Gorshkov, Optimal and secure measurement protocols for quantum sensor networks, Phys. Rev. A 97, 042337 (2018).

10. T. J. Proctor, P. A. Knott, and J. A. Dunningham, Multiparameter estimation in networked quantum sensors, Phys. Rev. Lett. 120, 080501 (2018).

11. S. Boixo, S. T. Flammia, C. M. Caves, and JM Geremia, Generalized Limits for Single-Parameter Quantum Estimation, Phys. Rev. Lett. 98, 090401 (2007).

12. M. D. Lang and C. M. Caves, Optimal Quantum-Enhanced Interferometry Using a Laser Power Source, Phys. Rev. Lett. 111, 173601 (2013).

13. Q. Zhuang, Z. Zhang, and J. H. Shapiro, Distributed quantum sensing using continuous-variable multipartite entanglement, Phys. Rev. A 97, 032329 (2018).

14. X. Guo, C. R. Breum, J. Borregaard, S. Izumi, M. V. Larsen, T. Gehring, M. Christandl, and J. S. Neergaard-Nielsen, U. L. Andersen, Distributed quantum sensing in a continuous variable entangled network, quant-ph/1905.09408.

15. S. Steinert, F. Dolde, P. Neumann, A. Aird, B. Naydenov, G. Balasubramanian, F. Jelezko, and J. Wrachtrup, High sensitivity magnetic imaging using an array of spins in diamond, Rev. Sci. Instrum. 81, 043705 (2010).

16. L. T. Hall, G. C. G. Beart, E. A. Thomas, D. A. Simpson, L. P. McGuinness, J. H. Cole, J. H. Manton, R. E. Scholten, F. Jelezko, J. Wrachtrup, S. Petrou, and L. C. L. Hollenberg, High spatial and temporal resolution wide-field imaging of neuron activity using quantum NV-diamond, Sci. Rep. 2 (2012).

17. L. M. Pham1, D .Le Sage, P. L. Stanwix, T. K. Yeung, D. Glenn, A. Trifonov, P. Cappellaro, P. R. Hemmer, M. D. Lukin, H. Park, A. Yacoby, and R. L. Walsworth, Magnetic field imaging with nitrogen-vacancy ensembles, New J. Phys. 13, 045021 (2011).

18. M. Seo, A. Adam, J. Kang, J. Lee, S. Jeoung, Q. H. Park, P. Planken, and D. Kim, Fourier-transform terahertz near-field imaging of one-dimensional slit arrays: mapping of electric-field-, magnetic-field-, and Poynting vectors, Optics Express 15, 11781 (2007).

19. T. Baumgratz, and A. Datta, Quantum Enhanced Estimation of a Multidimensional Field, Phys. Rev. Lett. 116, 030801 (2016).

20. P. C. Humphreys, M. Barbieri, A. Datta, and I. A. Walmsley, Quantum Enhanced Multiple Phase Estimation, Phys. Rev. Lett. 111, 070403 (2013).

21. J. Liu, X.-M. Lu, Z. Sun, and X.Wang, J. Phys. A 49, Quantum multiparameter metrology with generalized entangled coherent state, New J. Phys. A 49, 115302 (2016).

22. J.-D. Yue, Y.-R. Zhang, and H. Fan, Quantum-enhanced metrology for multiple phase estimation with noise, Sci. Rep. 4: 5933 (2014).

23. P. A. Knott, T. J. Proctor, A. J. Hayes, J. F. Ralph, P. Kok, and J. A. Dunningham, Local versus global strategies in multiparameter estimation, Phys. Rev. A 94, 062312 (2016).

24. C. N. Gagatsos, D. Branford, and A. Datta, Gaussian systems for quantum-enhanced multiple phase estimation, Phys. Rev. A 94, 042342 (2016).

25. M. A. Ciampini, N. Spagnolo, C. Vitelli, L. Pezzé, A. Smerzi, and F. Sciarrino, Quantum-enhanced multiparameter estimation in multiarm interferometers, $S c i$. Rep. 6: 28881 (2016).

26. K. Arai, C. Belthangady, H. Zhang, N. Bar-Gill, S. J. De-Vience, P. Cappellaro, A. Yacoby, and R. L. Walsworth, Fourier magnetic imaging with nanoscale resolution and 
compressed sensing speed-up using electronic spins in diamond, Nat. Nanotechnol. 10, 859 (2015).

27. A. Lazariev and G. Balasubramanian, A nitrogen-vacancy spin based molecular structure microscope using multiplexed projection reconstruction, Sci. Rep. 5: 14130 (2015).

28. P. Komar, E. M. Kessler, M. Bishof, L. Jiang, A. S. Sørensen, J. Ye, and M. D. Lukin, A quantum network of clocks, Nat. Phys. 10, 582 (2014).

29. M. N. Nabighian, V. J. S. Grauch, R. O. Hansen, T. R. Lafehr, Y. Li, J. W. Peirce, J. D. Phillips, and M. E. Ruder, The historical development of the magnetic method in exploration, Geophysics 70, 33ND (2005).

30. T. J. Wright, B. E. Parsons, and Z. Lu, Toward mapping surface deformation in three dimensions using InSAR, Geophys. Res. Lett. 31, L01607 (2004).

31. F. D. Stacey, The seismomagnetic effect, Pure Appl. Geophys. 58, 5 (1964).

32. D. Gatto, P. Facchi, F. A. Narducci, and V. Tamma, Distributed Quantum Metrology with a Single Squeezed-Vacuum Source, Phys. Rev. Research 1, 032024(R) (2019)

33. W. Ge, K. Jacobs, Z. Eldredge, A. V. Gorshkov, and M. Foss-Feig, Distributed Quantum Metrology with Linear Networks and Separable Inputs, Phys. Rev. Lett. 121, 043604 (2018).

34. C. Oh, C. Lee, S. H. Lie, and H. H. Jeong, Optimal Distributed Gaussian Sensing, quant-ph/1910.00823

35. W. P. Schleich, Quantum Optics in Phase Space (Wiley, 2001).

36. A. Ferraro, S. Olivares, and M. G. A. Paris, Gaussian states in continuous variable quantum information (Bibliopolis, Napoli, 2005). 